\section{ONOMÁVADIN}

Revista semestral de lingüística, filología y traducción
PONTIFICIA UNIVERSIDAD

\title{
El interés chileno por aprender y conservar el idioma mapudungun en Chile ${ }^{1}$
}

The Chilean Interest in the Conservation and Learning of the Native Language Mapudungun in Chile

\section{Jana Pfefferle}

Georg-August-Universität Göttingen

Alemania

\section{(c) $\bigodot_{\mathrm{BY}} \bigodot_{\mathrm{ND}}$}

Jana Pfefferle: Seminar für Romanische Philologie, Georg-August-Universität Göttingen, Alemania. | Correo electrónico: j.pfefferle@stud.uni-goettingen.de 


\section{Resumen}

El idioma indígena del pueblo mapuche en Chile, el mapudungun, está en peligro de extinción a causa de que su uso ha sido y es discriminado en la sociedad. Como medida para conservar el idioma, el gobierno chileno ha implementado programas para enseñar el mapudungun en algunos colegios con una alta tasa de niños mapuches. Pero las medidas tomadas no alcanzan para realmente conservar el idioma ni dan acceso a todos los chilenos con raíces indígenas a una educación bilingüe. El objetivo de este estudio fue la investigación del apoyo de la sociedad chilena para una educación bilingüe para todos los chilenos y para la conservación del idioma mapudungun. La opinión chilena fue investigada a través de una encuesta en línea, en la que se muestra la tendencia, por lo menos de algunos sectores de la sociedad, a apoyar la conservación y el aprendizaje del mapudungun en colegios chilenos, no solo por parte de niños y niñas mapuches, sino también por parte del resto.

Palabras clave: mapudungun; Chile; lenguas indígenas; educación bilingüe; actitudes lingüísticas.

\section{Abstract}

The native language Mapudungun of the Mapuche people in Chile is in danger of going extinct because its use has been and is being discriminated against in society. In order to preserve the language, the Chilean government has been implementing programs to teach it in Chilean schools with a high percentage of native students. However, these measures are not enough to stop the extinction of the language, nor do they provide bilingual education to all native children. The objec- tive of this study was to investigate the support of the Chilean people for a bilingual education including all Chilean children, as well as for the conservation of the native language Mapudungun. The Chilean opinion was investigated by means of an online survey which has shown that the interviewed Chileans support not only the conservation of Mapudungun but also the teaching of it in Chilean schools for all Chilean children.

Keywords: Mapudungun; Chile; native languages; bilingual education; language attitudes.

1 Este artículo se basa en la Tesis de Grado: Pfefferle, Jana (2013): La influencia léxica del idioma indigena mapdungun mediante contacto lingüístico en la variedad chilena del castellano y su percepción por la sociedad chilena. Georg-August-Universität Göttingen. 


\section{Introducción}

Según un estudio público en 2012 del Instituto de Investigación en Ciencias Sociales de la Universidad Diego Portales (UDP) en Chile, la mayoría de la sociedad chilena (87,3\%) piensa que en Chile hay mucha discriminación. Entre las formas de discriminación percibida se encuentra, entre ser homosexual y color de piel, ser mapuche. Además, $76,5 \%$ de la ciudadanía piensa que una persona en Chile puede llegar a ser muy discriminada por ser mapuche (UDP, 2012). Esa discriminación no solamente se refiere al pueblo mapuche y su cultura, sino también a su idioma, el mapudungun.

Durante siglos el gobierno chileno y parte de la sociedad chilena han discriminado el uso cotidiano del mapudungun, lo que hoy en día tiene como consecuencia una disminución de hablantes (Censo Chileno, 2012). Una investigación realizada por el Centro de Estudios Públicos en 2006 aportó la información de que en las regiones rurales un $17,5 \%$ de los mapuches usa el mapudungun cada día y un $15,9 \%$ lo habla con niños chicos en casa. Mientras, en las regiones urbanas, en las que según la misma encuesta vive el $69,4 \%$ de la población indígena, un 1,9\% de esta población habla diariamente mapudungun y un 2,4\% con niños chicos (Riedemann Fuentes, 2008 : 173). Estos datos indican que, a pesar de que el número de mapuches en Chile está aumentando, el número de mapuches que se comunican en mapudungun está disminuyendo, por lo que el idioma se encuentra en peligro de extinción (Zimmermann, 1999: 110-111).

Para evitar esta extinción inminente, es necesario implementar programas en colegios y en la sociedad, lo que solamente será posible con el apoyo del pueblo chileno en su conjunto. Pero, ¿cuál es la percepción del pueblo chileno con respecto al mapudungun y su conservación? El objetivo de este estudio es contribuir al análisis de esta percepción y para ello se han formulado dos hipótesis generales basadas en el contexto histórico y social: (I) Los chilenos consideran el mapudungun un idioma con un prestigio inferior al castellano y por ello no tienen interés en aprenderlo y (II) los chilenos quieren conservar el mapudungun para su patrimonio cultural. Afirmar o rechazar estas hipótesis mediante una investigación empírica permitiría el aumento o la adaptación de los programas de conservación para asegurar su eficacia máxima. Para alcanzar el objetivo de este estudio, se llevó a cabo una encuesta en línea con 237 participantes chilenos en 2013. El siguiente trabajo primero discute el contexto social del mapudungun y los programas establecidos para la conservación y el aprendizaje del idioma y a continuación presenta la metodología y los resultados de la encuesta. En la discusión se interpretan los resultados y su importancia de cara a la conservación del mapudungun.

\section{Contexto}

\subsection{La presencia y el rol actual del ma- pudungun en Chile}

Según Fishman (1967), la diglosia es un acuerdo social y solamente existe si el bilingüismo en un país está institucionalizado. En Chile, solamente existe un idioma oficial, el castellano, lo que muestra que no hay espacio para el mapudungun u otros idiomas indígenas en el ámbito público chileno (Riehl, 2004: 16-17). Aunque el mapudungun y los mapuches están reconocidos como pueblo hoy en día, el estado sigue con una actitud de indiferencia hacia el idioma indígena. En 2009, la Corporación Nacional de Desarrollo Indígena (CONADI) declaró que "salvando excepciones individuales las lenguas indígenas de Chile son asunto de indígenas" (CONADI, 2009: 17).

La discriminación histórica del pueblo mapuche y del mapudungun por parte del estado chileno es visible en el hecho de que hasta la implementación de la Ley Indígena el 5 de octubre de 1993 la existencia de los mapuches y su idioma no eran reconocidos oficialmente (Luna 
F., 2007: 21). En los colegios, el uso del mapudungun estuvo prohibido hasta 1994 con la excusa de que la asimilación de los pueblos indígenas a la cultura española no permite el uso de idiomas indígenas en los colegios (Espinosa Santos, 2008: 243, 246). Además, se quería evitar préstamos léxicos del mapudungun en el español chileno para conseguir un idioma chileno unido y estandarizado (Weinreich, 1977: 118). De esta manera, para asistir a los colegios estatales en Chile, lo que fue obligatorio para todos los chilenos a partir de 1920, los mapuches tuvieron que aprender el castellano (Ministerio de Educación de Chile [MINEDUC], 2008: 1). Por la falta de oportunidades y posibilidades futuras en Chile sin el conocimiento del castellano y por la necesidad de hablarlo en el ámbito público, el número de hablantes monolingües y bilingües del mapudungun está disminuyendo (Zimmermann, 1999: 110). Ya en 1966, Oroz se dio cuenta de que no había niños mapuches bilingües y de que, aunque lo entendían, pocos de ellos hablaban mapudungun a causa de que se usaba solamente el casteIlano en la vida cotidiana e, incluso, en el ámbito familiar (Oroz, 1966: 44). Hoy, solamente una minoría de los mapuches es bilingüe mientras casi no existen mapuches monolingües en mapudungun a pesar de que hace 100 años la mayoría de los mapuches no hablaba castellano (Salas, 1992: 43-44).

Según una estimación, en 2011 vivían 926.060 mapuches mayores de 14 años en Chile, de los cuales 60\% vivía en la Región Metropolitana (Repetto, 2011: 23). De ese 60\%, solamente $19,7 \%$ se declaró como bilingüe en español y mapudungun, mientras que el otro $80,03 \%$ solamente hablaba español. Aún más significativo es el hecho de que de ese $19,7 \%$ de hablantes bilingües, 40,09\% dijeron entender "un poquito", 34,1\% dijeron entender "casi todo" y solamente $25 \%$ dijeron entender "bastante" del mapudungun. Además, solamente $12,8 \%$ de los niños mapuches con lengua materna castellano aprendieron el mapudungun como segunda lengua (CONADI, 2009: 17-19). Otro estudio con 100 participantes mapuches mostró que, de ellos, un $84 \%$ no sabía hablar su idioma (Luna F., 2007: 57). Según la Encuesta de Caracterización Socioeconómica Nacional (CASEN) elaborada en 2006 por el Ministerio de Planificación, de los 925,005 chilenos que se autoidentificaron como pertenecientes al pueblo mapuche, solamente un 22,8\% declararó hablar o entender el mapudungun (Riedemann Fuentes, 2008: 173).

La falta de posibilidades para usar oficialmente el mapudungun en Chile y la discriminación histórica son responsables de la disminución del número de hablantes, lo que sitúa el mapudungun en riesgo de extinción (Espinosa Santos, 2008: 247)². Para impedir la extinción del mapudungun, es imprescindible tomar medidas para su conservación ahora, antes de que sea demasiado tarde.

\subsection{Programas recientes del gobierno chileno para la conservación y el apren- dizaje del mapudungun}

Uno de los primeros pasos hacia la conservación de los idiomas indígenas en Chile fue la implementación de la Ley Indígena en 1993 para reconocer los pueblos indígenas y - como parte importante de las raíces culturales chilenassus idiomas. Esta ley prometió protección, promoción y desarrollo de los pueblos indígenas y supuso el compromiso por parte del estado de apoyar la promoción de las tradiciones indígenas como parte del patrimonio cultural chileno (Luna F., 2007: 21; Repetto, 2011: 127). Además, la ley sugiere que se podrían usar los idiomas indígenas también en el ámbito social y en los colegios (Espinosa Santos, 2008: 243).

Con el Programa Intercultural Bilingüe (PIB), introducido como parte de la última reforma del

2 Hoy en día solamente se hablan tres de los 13 idiomas indígenas originales de Chile. Cinco idiomas ya están extinguidos y dos están a punto de extinguirse, el kawesquar o alcalufe y el yaghan o yámana (Espinosa Santos, 2008: 237-239). 
sistema educativo en 1996, debían ser integrados conocimientos indígenas a los planes de clase. Este programa, que intentó Ilevar la cultura indígena a las escuelas, no fue implementado de la manera esperada. Como consecuencia, varios grupos políticos, entre ellos el Grupo de Trabajo para los Pueblos Indígenas, se esforzaron en Ilevar la educación intercultural a los colegios en regiones con una alta población indígena (Saavedra Peláez, 2002: 213).

En 2003, el estado implementó la Ley General de Educación, que se centró en la educación intercultural y bilingüe. De ello resultó el Programa de Educación Intercultural Bilingüe (PEIB), que estableció que en escuelas con una alta tasa de estudiantes indígenas se debían enseñar las lenguas indígenas como asignatura. Además, los estudiantes no indígenas debían saber identificar y valorar las culturas indígenas (MINEDUC, 2010: 3-9).

En 2009 se aprobó el Decreto Supremo Nº 280 que hace obligatorio incorporar los idiomas mapudungun, rapa nui, aymara y quechua en colegios donde haya un porcentaje igual o mayor al 50\% de estudiantes con raíces indígenas. En la región de Los Ríos, que tiene un alto porcentaje de ciudadanos con ascendencia mapuche, aproximadamente 180 colegios municipales y particulares subvencionados empezaron implementar la enseñanza de mapudungun en el año escolar 2014. Para este fin, han sido formados treinta profesionales de la educación para llevar a cabo la enseñanza no solamente de la lengua mapuche, sino también de la cultura (MINEDUC, 2013). Además se publicaron las Guías Pedagógicas del Sector Lengua Indígena: Material de Apoyo Para La Enseñanza del Mapuzugun en 2010 para dar materiales de enseñanza y ayudar con la planificación de clases (MINEDUC, 2010).

El nuevo Plan de Estudios para los niveles entre $1^{\circ}$ y $4^{\circ}$ Básico publicado por MINEDUC en 2012 existe en dos formas. La primera no inclu- ye la asignatura de "Lengua Indígena”, mientras que la segunda incluye 152 horas anuales de "Lengua Indígena", con las horas tomadas de las otras asignaturas. El segundo plan es para colegios con un porcentaje alto de estudiantes con raíces indígenas, sin embargo, para colegios que tienen solamente una pequeña tasa de estudiantes indígenas se aplica el primer plan. Para los niveles escolares más avanzados todavía no está incluida la asignatura "Lengua Indígena”. En todo el país se está financiando la implementación del PEIB en 392 escuelas por parte del MINEDUC y el Banco Interamericano del Desarrollo (Riedemann Fuentes, 2008: 179).

En la Región Metropolitana, donde vive aproximadamente el $60 \%$ de los mapuches según una estimación de 2011 (Saavedra Peláez, 2002: 18), ningún colegio alcanza el porcentaje de niños con ascendencia indígena necesario para tener que impartir un idioma indígena. Además, en 2012 solamente unos 400 colegios participaron en el PEIB, aunque deberían ser aproximadamente 1600 en todo el país según el Decreto Supremo $N^{\circ} 280$ (Comunicaciones ANIDE, 2012). Mientras Riedemann Fuentes alerta de los peligros de una educación separada de los niños con ascendencia indígena3 ${ }^{3}$, pone enfásis en el hecho de que, para conseguir realmente una educación intercultural, hay que incluir a todo el alumnado chileno en la enseñanza de culturas indígenas, incluyendo la oferta de la asignatura "Lengua Indígena" para todos los alumnos que tengan interés (2008: 189).

La educación bilingüe no ha sido tenida en cuenta en otros planes impulsados por el gobierno, como, por ejemplo, el Plan de Araucanía 7, que tiene como objetivo que la Araucanía, la región más pobre de Chile, alcance los índices de desarrollo nacional en doce años (Gobierno de Chile, 2011). Con respeto a la educación, el plan solo contempla el "fortalecimiento de la enseñanza con pertinencia cultural mapuche” (Gobierno de Chile, 2011). 
Como la enseñanza bilingüe sí tiene el poder de frenar el proceso de extinción de idioma (Zimmermann, 1999: 140-142), es muy importante la implementación de la asignatura "Lengua Indígena" en todos los colegios chilenos. De hecho, el PEIB solamente podría detener la extinción del mapudungun en Chile si es implementado de manera extensa y da la oportunidad a todos de aprender y conservar su propio idioma (Saavedra Peláez, 2002: 212). Para eso, hay que tomar en cuenta que el idioma no es solamente un medio de comunicación, sino también el intermediario de cultura y tradición. Así, la extinción de un idioma trae consigo la extinción de una cultura (Zimmermann, 1999: 114-116, 118). El gobierno tiene que darse cuenta de que la política educacional y el PEIB forman una parte imprescindible tanto para la conservación y la supervivencia de las lenguas indígenas como para la conservación del patrimonio cultural chileno (Zimmermann, 1999: 140, 141)

\section{Estudio}

\subsection{La encuesta}

Entre el 1 de mayo y el 16 de junio de 2013 se llevó a cabo una encuesta en línea con el programa limesurvey para investigar la percepción de los chilenos del mapudungun y de la influencia del mismo idioma en la variedad chilena del castellano. La encuesta consistió en 15 preguntas que se enfocaron en el perfil demográfico de los participantes, su identificación con el habla chilena, la influencia léxica del mapudungun en el habla chilena y la percepción chilena del mapudungun. Relevante para el enfoque de este artículo es la investigación de la percepción de los participantes chilenos del mapudungun en relación con las hipótesis I y $1 \mathbf{I}^{4}$.

Las preguntas de investigación que se corresponden con las hipótesis mencionadas en la introducción son:
(I) ¿Tienen los participantes chilenos interés en aprender cualquier otro idioma?, ¿incluyen a este interés el idioma mapudungun? y capoyarían que los estudiantes chilenos aprendan el mapudungun como asignatura en los colegios?

(II) ¿Quieren conservar la lengua originaria mapudungun?

Las preguntas relevantes de la encuesta para el análisis de las hipótesis mencionadas fueron (1) “¿Le gustaría aprender otro idioma?”, (2) “¿Le gustaría aprender la lengua mapuche?”, (3) "¿Los estudiantes en Chile deben aprender el mapudungun (el idioma mapuche) en el colegio?” y (4) “¿Es importante que los niños mapuches aprendan su lengua (el mapudungun)?”. Las tres opciones a elegir como respuesta fueron "Sí", "No" y para las preguntas 2 y 3 también "Algunas palabras".

La encuesta fue difundida por correo electrónico y Facebook a contactos estudiantiles en un colegio subvencionado en Coquimbo y entre personas de la IV Región y la Región Metropolitana, así como entre académicos chilenos en todo el mundo con la petición de reenviar el enlace electrónico para que se distribuyese por el país.

La encuesta fue completada por 237 chilenos. De ellos, 47 participantes tienen entre 10-20 años, 89 participantes entre 21-30 años, 77 participantes entre 31-40 años, 19 participantes entre 41-50 años y 5 participantes más de 50 años. Como se puede ver en el mapa 1 , los participantes provienen mayoritariamente de la Región Metropolitana (40\%), Ia IV Región (18\%), Ia V (8\%), y la XIV Región (5\%). Algunas personas (17,74\%) respondieron la encuesta desde el extranjero. Las personas participantes en la encuesta no representan perfectamente la distribución de la población chilena, pero el porcentaje resulta 


\section{MAPA 1}

Lugar de residencia de las personas participantes viviendo en Chile (82.26\%)

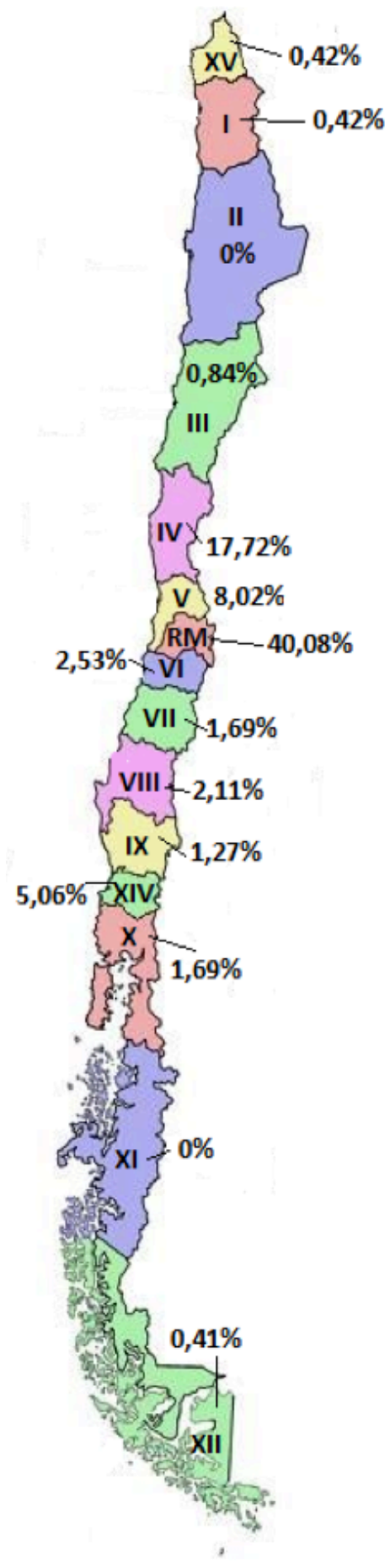

adecuado en el sentido de que, por ejemplo, también el $40 \%$ de la población chilena vive en la Región Metropolitana según el Censo 2002 (Censo Chileno, 2003).

Con respecto a la formación escolar de los participantes, más de un tercio tiene formación más allá de $4^{\circ}$ Medio. Mientras que un $26 \%$ (61) tiene un nivel de formación de $8^{\circ}$ Básico $04^{\circ}$ Medio, muchos de ellos son estudiantes que todavía no han terminado el colegio o la universidad, $33 \%$ son profesionales o técnicos de nivel superior, 30\% son licenciados de una universidad (Bachiller, Licenciado, Magister) y $11 \%$ tienen un doctorado. Por lo tanto, las personas participantes representan una parte de la población con un nivel de formación alto en comparación con la población chilenas

\subsection{Resultados}

Las respuestas a las preguntas sobre la percepción del mapudungun y la disposición para aprender el mismo idioma están resumidas en la tabla 1. La pregunta (1) "¿Le gustaría aprender otro idioma?" fue respondida por el 99\% de los participantes con "Sí". A la pregunta (2) "¿Le gustaría aprender la lengua mapuche?”, el 84\% (200) de las personas informantes respondieron con "Sí" o con "Algunas palabras" y el $\mathbf{1 6 \%}$ (37) con "No". La pregunta (3), "¿Los estudiantes en Chile deben aprender el mapudungun (el idioma mapuche) en el colegio?", obtuvo un "Sí" o un "Algunas palabras" por parte del 73\% (173) de los participantes y un "No" por parte del $27 \%$ (64). A propósito de los niños mapuches, el 95\% (226) de las personas informantes respondieron con "Si" a la pregunta (4) " ¿Es importante que los niños mapuches aprendan su lengua (el mapudungun)?".

5 Según el Censo Chileno de 1992, 42\% de la población chilena mayor de 15 años tiene como nivel de formación alcanzado $8^{\circ}$ Básico, $14 \% 4^{\circ}$ Medio, $26 \%$ una formación profesional y $18 \%$ un título de la universidad (Aedo l., 2000: 33). Hay que dar por hecho que esos números han cambiado en los últimos años. 


\section{TABLA 1}

Opinión sobre el mapudungun

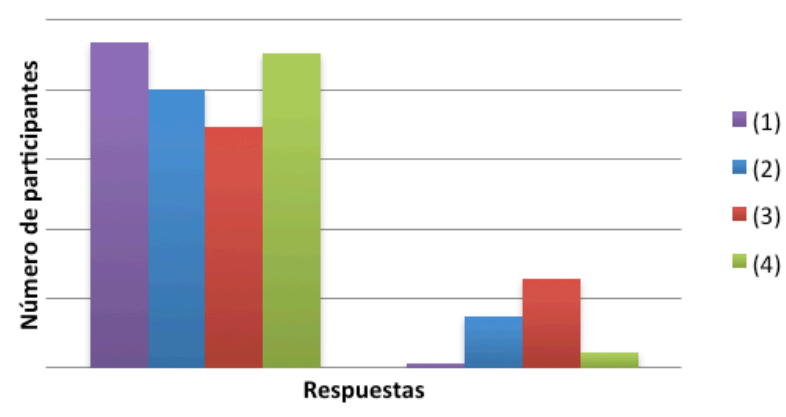

Respuestas a las preguntas:

(1) “¿Le gustaría aprender otro idioma?”,

(2) “¿Le gustaría aprender la lengua mapuche?”,

(3) “'Los estudiantes en Chile deben aprender el mapudungun (la lengua mapuche) en el colegio?” y

(4) “¿Es importante que los niños mapuches aprendan su lengua (el mapudungun)?”

\section{Discusión y conclusión}

La encuesta mostró que los chilenos informantes se muestran partidarios de conservar el mapudungun y de que este idioma indígena se pueda estudiar en el colegio. La primera hipótesis, es decir, que los chilenos consideran el mapudungun un idioma con un prestigio inferior al castellano y que por ello no tienen interés en aprenderlo, no se ve apoyada por los resultados de la encuesta, en la que se puede mostrar la tendencia ascendente en el interés por aprender el mapudungun por parte de las personas informantes. Por el contrario, la segunda hipótesis, es decir, que los chilenos quieren conservar la lengua mapuche para su patrimonio cultural, se puede confirmar en esta encuesta según los resultados.

A pesar de que la encuesta muestra claramente una actitud positiva hacia el mapudungun, hay que tomar en cuenta de que esta solamente llegó a una pequeña parte de la sociedad chilena y que no incluye a personas de todos los grupos demográficos chilenos. Mientras que todas las edades están representadas, no se ha podido encuestar a informantes de todas las regiones del país. La mayoría de los participantes proviene de la IV Región y de la Región
Metropolitana. El nivel de la formación académica también es muy alto, puesto que el 41\% de las personas participantes tienen un título universitario, lo que no es representativo de la población chilena. Para obtener resultados más representativos, se debería realizar otra encuesta con más participantes en todo el país y con representación de todos los grupos demográficos.

Como Riedemann Fuentes (2008), este artículo cuestiona por qué el estado está apoyando la enseñanza de lenguas indígenas y la cultura indígena exclusivamente en regiones donde hay una mayoría de niños con ascendencia indígena y no en todas las instituciones educacionales del país. También hay que considerar a los niños con ascendencia indígena que viven en regiones con una población indígena mínima y que deberían tener también el derecho a acceder a una educación pública en el idioma propio de su pueblo, como todos los niños indígenas, según Parte IV, artículo 15 de la Declaración de las Naciones Unidas (Sir, 2008: 23).

Si atendemos a los resultados de esta encuesta, el gobierno sí tendría apoyo social para expandir el PEIB a todo el país y no debería tardar en hacerlo. Además, es necesario complementar el PEIB con la promoción del uso de los idiomas indígenas en el ámbito público para también posibilitar el uso cotidiano de los idiomas y dar sentido a su aprendizaje. Esas medidas también funcionarían en contra de la discriminación hacia los idiomas indígenas en Chile y fortalecerían la integración, así como el conocimiento lingüístico y cultural de la población chilena.

\section{Bibliografía citada}

Aedo I., Cristian, 2000: "Evaluación y Recomendaciones de Política" [http://fen.uahurtado.cl/wpcontent/uploads/2010/07/inv125.pdf, fecha de consulta 4 de julio de 2013].

Comunicaciones ANIDE, 2012: "Situación y limitaciones de la Educación Intercultural 
Bilingüe" [http://libertadninos.wordpress. com/2012/10/23/situacion-y-limitaciones-de-laeducacion-intercultural-bilingue-2/, fecha de consulta 5 de mayo de 2014].

Corporación Nacional de Desarrollo Indígena (CONADI), 2009: Perfil sociolingüístico de lenguas mapuche y amara en la región metropolitana, Santiago de Chile: LOM Ediciones.

Espinosa Santos, Victoria, 2008: "Chile" en Azucena Palacios Alcaine (ed.): El español de América. Contactos lingüísticos en Hispanoamérica, Barcelona: Ariel, 237-254.

Fishman, Joshua, 1967: "Bilingualism With and Without Diglossia”, Journal of Social Issues 23/2, 29-38.

Gobierno de Chile, 2011: "Plan de Araucanía 7" [http://araucania7.cl/el-plan/, fecha de consulta 8 de mayo de 2014].

Instituto Nacional Estadisticas Chile, 2003: "Censo 2002 Resultados: Volumen I Población País-Región" [http://www.ine.cl/canales/usuarios/ce doconline/censos/pdf/censo_2002 _volumen_l. pdf, fecha de consulta 12 de febrero de 2015].

Instituto Nacional Estadísticas Chile, 2012: "Censo 2012" [http://www.censo.cl, fecha de consulta 24 de mayo de 2013].

LunA F., Laura, 2007: Un Mundo entre dos Mundos: Las relaciones entre el Pueblo Mapuche y el Estado Chileno desde la perspectiva del desarrollo y de los cambios socioculturales, Santiago, Chile: Ediciones Universidad Católica de Chile.

Ministerio de Educación de Chile (MINEDUC), 2008: "12 años de escolaridad obligatoria en Chile” [http:// www.educarchile.cl/Userfiles/P0001/File/12_ anosEscolaridad Obligatoria.pdf, fecha de consulta 1 de julio de 2013].

Ministerio de Educación de Chile (MINEDUC), Programa de Educación Intercultural Bilingüe, 2010: "Guías pedagógicas del sector lengua indígena: Material de apoyo para la enseñanza del Mapu- zugun" [http://www.unicef.cl/unicef/public/ar chivos_documento/328/mapuche.pdf, fecha de consulta 26 de abril de 2013].

Ministerio de Educación de Chile (MineduC), 2012: "Currículum nacional. Planes de Estudios" [http://www.mineduc.cl/index5_int.php?id_ portal $=47 \& i d_{-}$contenido=17116\&id_ seccion $=3264 \& c=346$, fecha de consulta 21 de marzo de 2014].

Ministerio de Educación de Chile (MineduC), 2013: "Mineduc certificó a educadores tradicionales en programa de estudio de $4^{\circ}$ básico del Sector de Lengua y Cultura Mapuche" [http:// www.losrios.mineduc.cl/index2.php?id_ contenido=27341\&id_portal=34\&id_sec cion $=2263$, fecha de consulta 29 de diciembre de 2013].

Oroz, Rodolfo, 1966: La lengua castellana en Chile, Universidad de Chile, Santiago de Chile: Editorial Universitaria.

Repetto, Maxim, 2011: Políticas Indigenistas en el Cono Sur: Chile y Argentina contra el Pueblo Mapuche, siglos XIX y XX, Saarbrücken: Editorial académica española.

Riedemann Fuentes, Andrea, 2008: "La educación intercultural bilingüe en Chile: campliación de oportunidades para alumnos indígenas?", Indiana 25, 169-193.

Rient, Claudia Maria, 2004: Sprachkontaktforschung: Eine Einführung, Tübingen: Narr Studienbücher.

Saavedra Peláez, Alejandro, 2002: Los Mapuches en la sociedad chilena actual, Santiago de Chile: Editorial LOM.

Salas, Alberto, 1992: El Mapuche o Araucano: Fonología, gramática y antología de cuentos, Madrid: Editorial Mapfre.

SIR, Jorge, 2009: "Una aproximación a la educación intercultural bilingüe en el sistema escolar chileno", Docencia 37, 19-25. 
Universidad Diego Portales, Instituto de Investigación en Ciencias Sociales, 2012: "Elites Políticas Discriminación y diversidad" [http://estudios. anda.cl/estudio.aspx?id_estudio=4525, fecha de consulta: 8 de mayo de 2014].

WeinReich, Uriel, 1977: Sprachen in Kontakt: Ergebnisse und Probleme der Zweisprachigkeitsforschung, München: C.H. Beck'sche Verlagsbuchhandlung.

Zimmermann, Klaus, 1999: Política del lenguaje y planificación para los pueblos amerinidios: Ensayos de ecología lingüística, Madrid: Iberoamericana. 